\title{
Reviewers for Journal of Hematopathology in 2019
}

Published online: 27 January 2020

(C) Springer-Verlag GmbH Germany, part of Springer Nature 2020

The following people donated their time and expertise as peer-reviewers of manuscripts submitted to the Journal of Hematopathology in 2019. The Journal thanks them all for their invaluable contributions. An asterisk follows the name of those reviewers who evaluated three or more manuscripts in 2019.

Patrick Adam, MD

Archana Agarwal, MD

Nidhi Aggarwal, MD

Catalina Amador, MD

Emanuele Angelucci, MD

Mary Ann Thompson Arildsen, MD, $\mathrm{PhD}$

Aditya Badheka, MBBS, MS

Nathanael G. Bailey, MD

Carlos Barrionuevo, MD, MSc

Daniel Boyer, MD, $\mathrm{PhD}$

Noah A. Brown, MD

Carlos Bueso-Ramos, MD

Alexander C. L. Chan, MD

Shweta Chaudhary, MD

Ding-Bao Chen, MD

Dong Chen, MD, PhD

April Chiu, MD

Sarah Choi, MD, PhD

Elizabeth Courville, MD

Paola dal Cin, $\mathrm{PhD}$

Daphne de Jong, MD, PhD

Laurence de Leval, MD, PhD

Rajan Dewar, MD, PhD

Amy S. Duffield, MD, PhD

Mohsen Saleh Elalfy, MD, PhD

Andrew L. Feldman, MD, PhD*

Falko Fend, MD

Judith A. Ferry, MD

John K. Frederiksen, MD, PhD*

Julia T. Geyer, MD

Juan Gomez-Gelvez, MD*

Jerald Z. Gong, MD

John R. Goodlad, MB ChB, MD

Patricia T. Greipp, DO

Timothy M. Hanley, MD, PhD
Marian H. Harris, MD, PhD

Robert P. Hasserjian, MD

Rong He, MD

K.M. Hebeda, MD, PhD

Nahla M. Heikal, MD

Bo Hong, MD

Pedro Horna, MD

Matthew T. Howard, MD

James D. Hoyer, MD

Sarika Prakash Jain, MD

Jesse Jaso, MD

Ridas Juskevicius, MD

Rina Kansal, MD

Kristin H. Karner, MD*

David F. Keren, MD

Rebecca L. King, MD

Matthew Klairmont, MD

Wolfram Klapper, MD

Monika Klimkowska, MD, PhD

Scott Charles Kogan, MD

Alexandra Kovach, MD*

Heather Leitch, MD, PhD

Lorenzo Leoncini, MD

Megan Lim, MD, PhD

Yen-Chun Liu, MD, PhD

Sanam Loghavi, MD

Abner Louissaint, MD, PhD

William R. Macon, MD*

Carmen Lome Maldonado, MD

Daniel S. Martig, MD

Maurizio Martini, MD, $\mathrm{PhD}$

Emily F. Mason, MD, PhD

Matthew J. Maurer, MS

Penelope Anne McKelvie, MBBS

Rodney R. Miles, MD, PhD

Roberto N. Miranda, MD*
Nathan Montgomery, MD, PhD

Erika Moore, MD

Elizabeth A. Morgan, MD

Karen A. Moser, MD

Claudio A. Mosse, MD, PhD

Pawel Mroz, MD, PhD*

Carlos Murga-Zamalloa,

Megan Okumoto Nakashima, MD*

Valentina Nardi, MD*

Kikkeri N. Naresh, MD

Jadee Neff, MD, PhD

Chi Young Ok, MD

Horatiu Olteanu, MD, PhD

Stacey O'Neill, MD, PhD

German Ott, MD

Cristina Papayannidis, MD

Jess F. Peterson, MD

Rajiv K. Pruthi, MBBS

Phil Raess, MD, PhD

Bryan Rea, MD

Karen L. Rech, MD

Eduardo Rego, MD, PhD

Kaaren K. Reichard, MD

Erica F. Reinig, MD

Heesun J. Rogers, MD, PhD

Flavia G. Rosado, MD

Terezinha Marques Salles, $\mathrm{PhD}$

Adam Seegmiller, MD, PhD

William Selove, MD

Aaron C. Shaver, PhD, MD

Min Shi, MD, PhD

Imran N. Siddiqi, MD, PhD

Kristi Johnson Smock, MD

Fernando A. Soares, MD, PhD

Soo Yong Tan, MBBS*

Zhenya Tang, MD, PhD 
Reha Toydemir, MD, $\mathrm{PhD}^{*}$ Jenny Turner, MBBS

Alexandar Tzankov, MD

Chris van Vliet, MBCHB
José Vassallo, MD, $\mathrm{PhD}$

Laura Wake, MD

Wei Wang, MD, PhD

Olga Weinberg, MD
Adam J. Wood, DO, MS

Rena R. Xian, MD

Jie $\mathrm{Xu}, \mathrm{MD}, \mathrm{PhD}$

Publisher's note Springer Nature remains neutral with regard to jurisdictional claims in published maps and institutional affiliations. 